\title{
Neural correlates and predictors of subjective cognitive decline in patients with Parkinson's disease
}

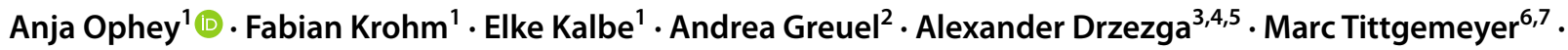 \\ Lars Timmermann $^{2,8} \cdot$ Frank Jessen $^{4,7,9} \cdot$ Carsten Eggers $^{2,8} \cdot$ Franziska Maier $^{9}$
}

Received: 18 April 2021 / Accepted: 4 November 2021 / Published online: 25 November 2021

(c) The Author(s) 2021

\begin{abstract}
Background Subjective cognitive decline (SCD) may occur very early in the course of Parkinson's disease (PD) before the onset of objective cognitive decline. Data on neural correlates and determinants of SCD in PD are rare.

Objective The aim of the present study was to identify neural correlates as well as sociodemographic, clinical, and neuropsychological predictors of SCD in patients with PD.

Methods We retrospectively analyzed 30 patients with PD without cognitive impairment ( $23 \%$ female, $66.90 \pm 7.20$ years, UPDRS-III: $19.83 \pm 9.29$ ), of which $n=12$ patients were classified as having no SCD (control group, PD-CG) and $n=18$ as having SCD (PD-SCD). Neuropsychological testing and 18-fluoro-2-deoxyglucose positron emission tomography (FDGPET) were conducted. SCD was assessed using a questionnaire covering multiple cognitive domains.

Results SCD subscores differed significantly between PD-CG and PD-SCD and correlated significantly with other scales measuring related concepts. FDG-PET whole-brain voxel-wise regression analysis revealed hypometabolism in middle frontal, middle temporal, and occipital areas, and the angular gyrus as neural correlates of SCD in PD. Next to this hypometabolism, depressive symptoms were an independent significant determinant of SCD in a stepwise regression analysis (adjusted $R^{2}=50.3 \%$ ).

Conclusion This study strengthens the hypothesis of SCD being an early manifestation of future cognitive decline in PD and, more generally, early pathological changes in PD. The early identification of the vulnerability for future cognitive decline constitutes the basis for successful prevention and delay of this non-motor symptom.
\end{abstract}

Keywords Parkinson disease $\cdot$ Subjective cognitive decline $\cdot$ Neural correlates $\cdot$ Positron emission tomography $\cdot$ Predictors

Anja Ophey and Fabian Krohm contributed equally; Carsten

Eggers and Franziska Maier contributed equally

Anja Ophey

anja.ophey@uk-koeln.de

1 Department of Medical Psychology I Neuropsychology $\&$ Gender Studies, Center for Neuropsychological Diagnostic and Intervention (CeNDI), Faculty of Medicine and University Hospital Cologne, University of Cologne, Kerpener Str. 68, 50937 Cologne, Germany

2 Department of Neurology, University Hospital of Marburg, Marburg, Germany

3 Department of Nuclear Medicine, Faculty of Medicine and University Hospital Cologne, University of Cologne, Cologne, Germany

4 German Center for Neurodegenerative Diseases (DZNE), Bonn-Cologne, Germany
5 Institute of Neuroscience and Medicine (INM-2), Molecular Organization of the Brain, Forschungszentrum Jülich, Jülich, Germany

6 Max Planck Institute for Metabolism Research, Cologne, Germany

7 Excellence Cluster On Cellular Stress Responses in Aging-Associated Diseases (CECAD), University of Cologne, Cologne, Germany

8 Center for Mind, Brain and Behavior - CMBB, Universities of Marburg and Gießen, Marburg, Germany

9 Department of Psychiatry and Psychotherapy, Faculty of Medicine and University Hospital Cologne, University of Cologne, Cologne, Germany 


\section{Introduction}

Subjective cognitive decline (SCD) describes a self-perceived, subjective deterioration in potentially different cognitive domains in the absence of objective cognitive impairment [1]. Subjective deterioration in the memory domain is being discussed to occur at late preclinical stage in Alzheimer's disease (AD) before the onset of mild cognitive impairment (MCI) and dementia [AD; 1]. SCD, especially in the memory domain, was identified as a risk factor for developing objective cognitive impairment [2, $3]$ and accelerates time to conversion to objective cognitive impairment [3]. Even though the construct of SCD gained increasing interest in the past decade, heterogeneous instruments predominantly focusing on the memory domain are adopted to assess SCD [4], and recommendations on SCD research criteria in the AD context just emerged [5].

Cognitive decline is a common non-motor symptom in Parkinson's disease (PD) [6]. Similarly to patients with $\mathrm{AD}, \mathrm{SCD}$ might constitute a possible precursor of objective cognitive decline in patients with PD [7]. For example, longitudinal studies show significantly higher incidences of PD-MCI and PD dementia for patients with PD and SCD at baseline compared to patients without SCD at baseline [8-10]. In the context of PD, it seems especially reasonable to focus on a broader construct of $\mathrm{SCD}$, as cognitive decline in PD is highly heterogeneous: executive functions, working memory, and attentional functions are among the earliest and most frequently impaired cognitive domains, but memory, visuo-cognitive, and language impairments are observed as well [11-14]. Therefore, the SCD assessment in PD should be based on a broad assessment of cognitive complaints across various cognitive domains. So far, SCD in PD is assessed by a broad range of measures, and there is little consistency between SCD operationalizations [7].

To gain a full understanding of SCD in PD, research on neural correlates and sociodemographic, clinical, and neuropsychological determinants and predictors of SCD is essential. These determinants, as well as neural correlates of SCD, however, have rarely been investigated in $\mathrm{PD}$, and existing studies focused on SCD in the memory domain only. Most of the studies investigating neural correlates of SCD in PD so far categorized their patients with one single question focusing on memory complaints [e.g., "Do you have any memory-related problems?", 15] and did not use validated SCD assessments.

In normal aging, especially associations of SCD with depressive symptoms and an interplay with objective cognitive functioning have been demonstrated [16]. Furthermore, possible determinants of SCD include demographic variables (e.g., female sex, older age), specific objective cognitive functions (e.g., global cognition, executive functions, language, processing speed), psychological factors (e.g., stress, anxiety), and personality characteristics (e.g., neuroticism) [16-20]. For PD, only anxiety has been identified as a highly significant and independent determinant of SCD so far, whereas age, disease severity, and functioning in instrumental activities of daily living did correspond with SCD [21].

Neural correlates of SCD in the AD context were mainly found in fronto-temporal, temporo-medial, and occipital regions [e.g., 22-25]. Considering morphometric neural correlates of SCD in PD assessed with MRI, cortical thinning and decreased grey matter density was found in the dorsolateral prefrontal, orbitofrontal, medial frontal, and parahippocampal cortices, anterior cingulate, and angular gyrus in patients with PD and SCD in the memory domain compared to $\mathrm{PD}$ patients without SCD $[9,15]$. Those findings are underlined by a SPECT study [26] demonstrating decreased perfusion in the thalamus and the anterior cingulate as well as in medial frontal and inferior temporal regions.

The objective of the current study was to identify sociodemographic, clinical, and neuropsychological determinants as well as functional neural correlates of SCD in patients with PD assessed with 18-fluoro-2-deoxyglucose positron emission tomography (FDG-PET) which have, to the authors' best knowledge, not been investigated in combination so far.

\section{Methods}

\section{Study sample}

For this study, a subsample of patients with PD from two studies at the University Hospital of Cologne was used. For details on the objective and recruitment procedure, please refer to Ruppert et al. [27] and Hammes et al. [28]. Both studies were approved by the ethics committee of the medical faculty of the University of Cologne, Germany (approval numbers 12-265 and 15-325). Every subject provided informed consent to participate in the study. The authors assert that all procedures contributing to this work comply with the ethical standards of the relevant national and institutional committees on human experimentation and with the Helsinki Declaration of 1975, as revised in 2008.

For the current analyses, inclusion criteria were (i) the diagnosis of PD according to UK brain bank criteria; (ii) the availability of FDG-PET imaging data; (iii) the availability of neuropsychological data including the modified and extended version of the Subjective Memory Impairment Questionnaire, the SCD-Q [29, 30]; (iv) the exclusion of 
PD-MCI or PD dementia according to level-II diagnostic criteria; and (v) the exclusion of other diseases affecting brain functions, e.g., operationalized by the intake of any centrally active medication such as antidepressant/ anxiolytics beyond the antiparkinsonian dopaminergic medication (which itself had to be stable for the last four weeks prior to and throughout study participation). These inclusion criteria were met by 30 patients (for details, see Flow Chart in Supplementary Material Fig. 1).

\section{Clinical aspects}

Clinical characteristics such as disease duration and the levodopa equivalent daily dose [LEDD; 31$]$ were recorded. Motor symptom severity was measured with the Unified Parkinson's Disease Rating Scale part 3 [UPDRS-III; 32] in ON- and OFF-state [27]. Depressive symptoms were examined using the Beck Depression Inventory 2 [BDI-II; 33].

\section{Neuropsychological assessment}

The neuropsychological assessment took place in the medication ON-state within 2 days of the FDG-PET scan.

\section{Subjective cognitive decline}

To assess SCD, a modified and extended version of the SCD-Q [29, 30] was used to cover a broader spectrum of cognitive functions that can be affected in PD. It assesses SCD over six cognitive domains: memory, attention, language, executive functions, visuo-cognitive skills, and social cognition. First, the patients have to answer a dichotomous question ("yes or no") on whether they experience worsening in the respective domain. Second, if present, concerns and worries regarding this worsening are assessed on a 3-point Likert-scale ("no" $=0$, "sometimes" =1, "yes" =2). Third, for each domain (irrespective of the answer to the first global question), questions about experiencing cognitive decline in specific daily situations have to be answered on a 4-point Likertscale from "never" $=0$ to "always" $=3$. Summarizing, three scores can be created: (i) subjectively impaired cognitive domains (SCD-D, 0-6 points), (ii) presence of worries regarding the subjectively impaired cognitive domains (SCD-W, 0-12 points), and (iii) specific cognitive concerns in daily life situations (SCD-S, 0-60 points).

To assess SCD-related concepts and to validate our concept of SCD, additional questionnaires were used. The first additional measure was the informant-rated version of the Everyday Cognition Questionnaire (ECog) containing 39 items [34]. For each item, the informant has to compare the patient's current state with the state 10 years prior and indicate whether the variable is unchanged or has worsened on a four-point-scale. The items are related to memory, language, visuo-cognitive functions, planning, organization, and attention. For evaluation, the sum score (maximum of 156 points) is divided by the number of items (maximum of 39 items) resulting in a score between 1 and 4 [34]. The second measure was the Cognitive Failures Questionnaire (CFQ) that is designed as self- and informant-reported questionnaire [35]. The CFQ contains 25 items indicating the frequency with which people experience cognitive failures in the past 6 months on a five-point-scale. Thus, maximum score is 100 points with lower scores indicating a lower frequency of cognitive failures [35]. Thirdly, the Dysexecutive Questionnaire (DEX), a subtest from the Behavioral Assessment of the Dysexecutive Syndrome, was used [36]. The DEX was designed to assess possible behavioral changes as a result of a dysexecutive syndrome. Both the self- and informant-rated version were used containing 20 items each that are rated in terms of frequency on a five-point-scale resulting in a total score ranging from 0 to 80 , with lower scores indicating less problems with executive functioning [36].

For group comparisons in the present study, the presence of SCD is defined as experienced worsening in at least two domains accompanied by at least partially ("sometimes") present worries. Following these criteria, $n=12$ patients were classified as having no SCD (PD control group; PD-CG) and $n=18$ as having SCD (PD-SCD). For the investigation of the relationship between SCD and SCD-related concepts, neural correlates of SCD, and other determinants of SCD, however, we used the SCD-S as a continuous measure within the analyses.

\section{Cognition}

As cognitive screening tools, the Mini-Mental State Examination [MMSE; 37] and the Parkinson Neuropsychometric Dementia Assessment [PANDA; 38] were used. To assess specific cognitive domains, additional neuropsychological tests were applied; that were also used to exclude the presence of objective cognitive impairment (PD-MCI or PD dementia). For the operationalization of Level-II diagnostic criteria for PD-MCI [39], the cut-off for impaired test performance was set at $\leq-1.5$ standard deviations according to published normative data. If two or more tests were impaired across the five assessed cognitive domains, the patient was excluded from the present analyses due to present objective cognitive impairment that might confound the SCD assessments. The cognitive screening tools were not relevant for excluding the presence of objective cognitive impairment. For further details on neuropsychological tests and their assignment to a cognitive domain, see Supplementary Material Table 1. 


\section{FDG-PET imaging data acquisition and pre-processing}

FDG-PET was performed under standard conditions with an average dose of $185 \mathrm{MBq}$ FDG on a 24-detector ring highresolution research tomograph (ECAT HRRT, Siemens) and preprocessed in SPM12 (http://www.fil.ion.ucl.ac.uk/spm/ software/spm12) running on MATLAB R2018a (The MathWorks, Inc.). Image acquisition procedure and preprocessing have previously been described in detail [27, 40]. FDG-PET acquisition was conducted in the medication OFF-state after at least 12-h discontinuation of levodopa, amantadine and MAO-inhibitors, and 72-h withdrawal from dopamine agonists. For the current analysis, normalized image dimensions were 91/109/91 voxels $(\mathrm{x} / \mathrm{y} / \mathrm{z})$ sized $2 \times 2 \times 2 \mathrm{~mm}$.

\section{Statistical analyses}

Statistical analyses were performed using SPSS 26 for Mac unless indicated otherwise. All data were tested for normal distribution with Shapiro-Wilk tests and for homogeneity of variances with Levene-tests. Comparisons of demographic, clinical, and cognitive variables between PD-CG and PD-SCD were calculated using independent sample $t$ tests, Mann-Whitney $U$ tests, or $\chi^{2}$ tests as appropriate. As effect sizes, Cohen's $d$ is reported for $t$ tests and $r$ for Mann-Whitney $U$ tests, indicating small $(d \geq 0.2 ; r \geq 0.1)$, moderate $(d \geq 0.5 ; r \geq 0.3)$, or strong $(d \geq 0.8 ; r \geq 0.5)$ effects. Spearman correlations were computed between the SCD-Q subscores (SCD-D, SCD-W, SCD-S) and the scores of questionnaires assessing related concepts, i.e., ECog, CFQ, and DEX.

FDG-PET correlates of SCD were analyzed in SPM12 by whole-brain voxel-wise regression with the SCD-S subscale of the SCD-Q, using age, sex, UPDRS-III OFF-state, and BDI-II scores as covariates, to assess the correlations between the specific concerns of SCD and FDG-metabolism in patients with PD. We chose the regression approach over a group comparison as we aimed to assess SCD as a continuous measure and no established criteria to categorize patients to PD-CG and PD-SCD were available. The initial threshold was set at $p \leq 0.01$ with a cluster extent threshold of 100 voxels. Results were considered significant at $p \leq 0.05$ uncorrected on cluster level. Although this is a less-conservative criterion, it is commonly used in studies investigating neural correlates of SCD and/or MCI in PD [9, 15, 26]. For anatomical identification of the local peaks, the WFU-PickAtlas Tool 2.4 (http://fmri.wfubmc.edu/software/pickatlas) was used to transform the coordinates to regions of the Automated Anatomical Labeling atlas (AAL).

In addition, the mean FDG uptake value in a region of interest (ROI) including fronto-medial, temporo-medial, and parieto-occipital areas was extracted using MarsBaR (http://marsbar.sourceforge.net). The ROI was defined based on previous literature on neural correlates of SCD in PD and AD. For specific regions included in the ROI, see Supplementary Material Table 2. The metabolism value of this ROI as well as age, sex, education, UPDRS-III ON-state, BDI-II, and PANDA were integrated into stepwise regression analyses to evaluate possible determinants of SCD in PD patients. Note, that we used UPDRS-III in OFF-state as covariate in the whole-brain voxel-wise regression analysis and in $\mathrm{ON}$-state as possible determinant in the stepwise regression analysis. With this differentiation, the medication state corresponded between the UPDRS-III assessment and the FDG-PET acquisition and neuropsychological testing, respectively.

\section{Results}

\section{Sample characteristics}

Sociodemographic and clinical characteristics as well as the overall cognitive state of the patients are displayed in Table 1. On average, patients were $66.90 \pm 7.20$ years old with a median PD duration of 3.5 years. PD-CG $(n=12)$ and PD-SCD $(n=18)$ did not differ significantly in sociodemographic variables, clinical characteristics, their global cognitive status, and further cognitive assessments.

\section{Subjective cognitive decline (SCD)}

In comparison to PD-CG, PD-SCD descriptively showed higher scores in all subscores of the SCD-Q and questionnaires assessing SCD related constructs (Table 1). The differences reached significance for all subscores of the SCD-Q (SCD-D: $U=4.00, p<0.001, r=0.82$; SCD-W: $U=4.50$, $p<0.001, r=0.81$; SCD-S: $t(28)=-4.69, p<0.001$, $d=1.75)$, the $\mathrm{ECog}(U=23.00, p<0.001, r=-0.62)$, and the self-rated version of both CFQ $(t(28)=-3.11, p=0.04$, $d=1.16)$ and $\operatorname{DEX}(t(28)=-3.30, p=0.003, d=1.23)$.

Spearman correlations between the SCD-Q subscores and further SCD related variables are reported in Table 2. As only the subscore SCD-S was further used in the FDGPET analysis and the stepwise regression analysis, here we exemplary outline further details of the correlations for the SCD-S subscore. The SCD-S correlated strongly and highly significantly with the self-rated version of the CFQ $(r=0.59, p=0.001)$ and the self-rated version of the DEX $(r=0.62, p<0.001)$. Weak but significant correlations were observed with the ECog $(r=0.44, p=0.018)$. Correlations between SCD-S and the informant-rated versions of both CFQ $(r=0.28, p=0.157)$ and DEX $(r=0.10, p=0.615)$ did not reach statistical significance. 


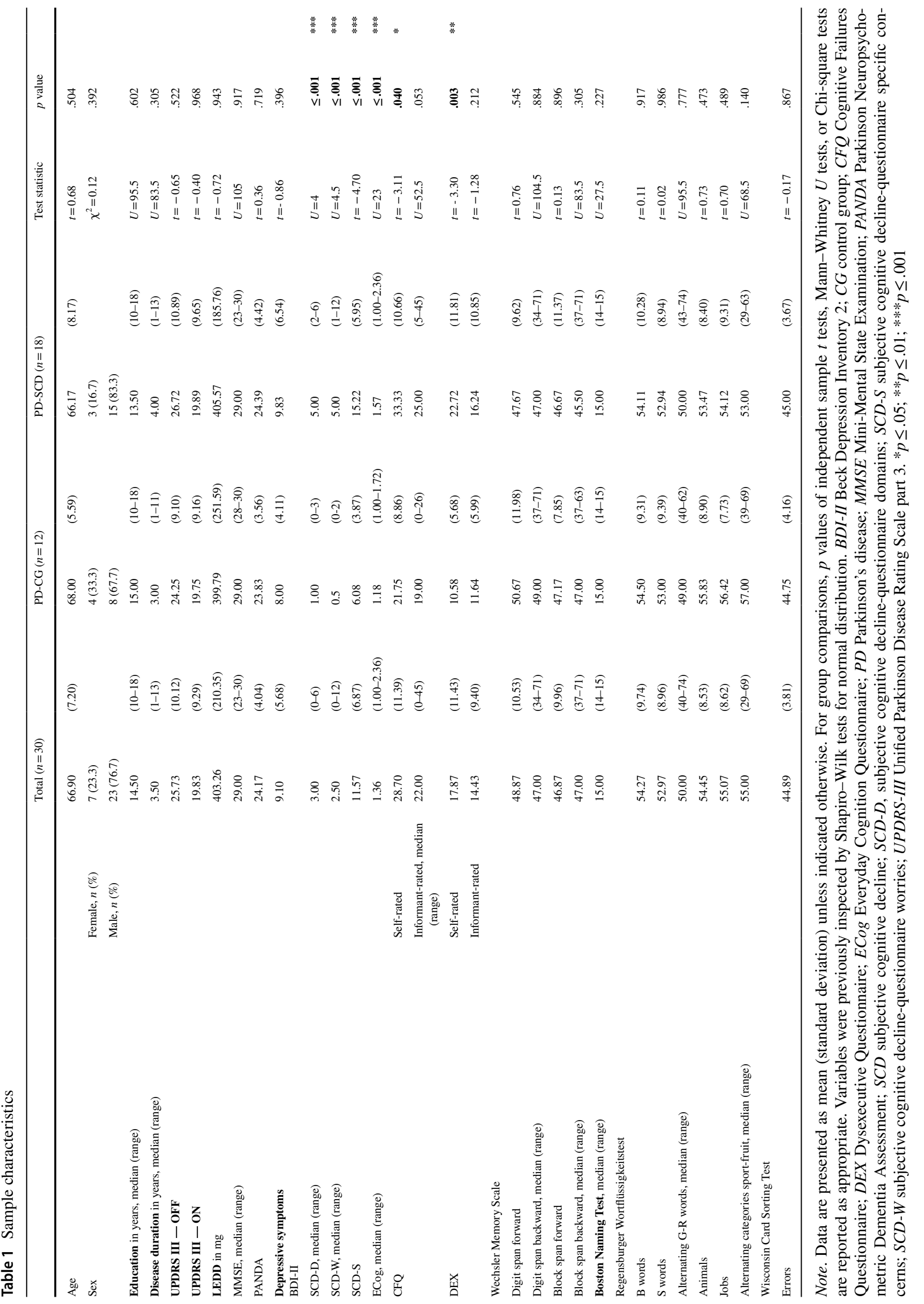


Table 2 Spearman correlations between subjective cognitive decline and related concepts

\begin{tabular}{|c|c|c|c|c|c|c|c|c|}
\hline & SCD-D & SCD-W & SCD-S & ECog & CFQ-S & CFQ-I & DEX-S & DEX-I \\
\hline SCD-D & 1 & $.915^{\star * *}$ & $.848^{* * *}$ & $.544^{* *}$ & $.454^{*}$ & .367 & $.535^{\star *}$ & .196 \\
\hline SCD-W & & 1 & $.818^{* * *}$ & $.563^{\star *}$ & $.515^{*}$ & $.422^{*}$ & $.618^{\star \star *}$ & .305 \\
\hline SCD-S & & & 1 & $.436^{*}$ & $.586^{\star *}$ & .275 & $.621^{\star \star *}$ & .099 \\
\hline ECog & & & & 1 & $.398^{*}$ & $.638^{\star * *}$ & $.479^{\star *}$ & $.511^{* *}$ \\
\hline CFQ-S & & & & & 1 & .323 & $.906^{\star * *}$ & .232 \\
\hline CFQ-I & & & & & & 1 & $.399^{*}$ & $.787^{\star * *}$ \\
\hline DEX-S & & & & & & & 1 & .228 \\
\hline DEX-I & & & & & & & & 1 \\
\hline
\end{tabular}

Note. CFQ-I Cognitive Failures Questionnaire informant-rated version; $C F Q-S$ Cognitive Failures Questionnaire self-rated version; $D E X-I$ Dysexecutive Questionnaire informant-rated version; $D E X-S$ Dysexecutive Questionnaire self-rated version; ECog Everyday Cognition Questionnaire; $S C D-D$ subjective cognitive decline-questionnaire domains; $S C D$ - $S$ subjective cognitive decline-questionnaire specific concerns; $S C D$ - $W$ subjective cognitive decline-questionnaire worries

${ }^{*} p \leq .05 ; * * p \leq .01 ; * * * p \leq .001$

\section{Neural correlates of SCD}

The whole brain voxel-wise regression analysis between FDG-metabolism and SCD-S score is presented in Table 3 and visualized in Fig. 1. Patients with higher SCD-S scores showed decreased metabolism in the following regions: right angular gyrus $\left(p=0.002, k_{\mathrm{e}}=1223\right)$, bilateral middle temporal gyrus (left: $p=0.026, k_{\mathrm{e}}=570$; right: $\left.p=0.009, k_{\mathrm{e}}=841\right)$, bilateral occipital regions including right precuneus $\left(p=0.012, k_{\mathrm{e}}=766\right)$, and left calcarine sulcus $\left(p=0.001, k_{\mathrm{e}}=1594\right)$, left middle frontal gyrus $\left(p=0.044, k_{\mathrm{e}}=448\right)$ including the orbital part $\left(p=0.043, k_{\mathrm{e}}=455\right)$. Higher SCD-S scores did not correlate with increased metabolism in any region.
Fig. 1 Whole brain voxel-wise regression analysis between FDG uptake and SCD-S score. Red-yellow maps illustrate negative correlation between FDG uptake and SCD-S score indicating hypometabolism in these areas. A 3D render view B 2D axial slices. L, left; P, posterior; R, right. Color bar indicates $t$ values. Slices and 3D display were constructed with MRIcroGL, neurological display
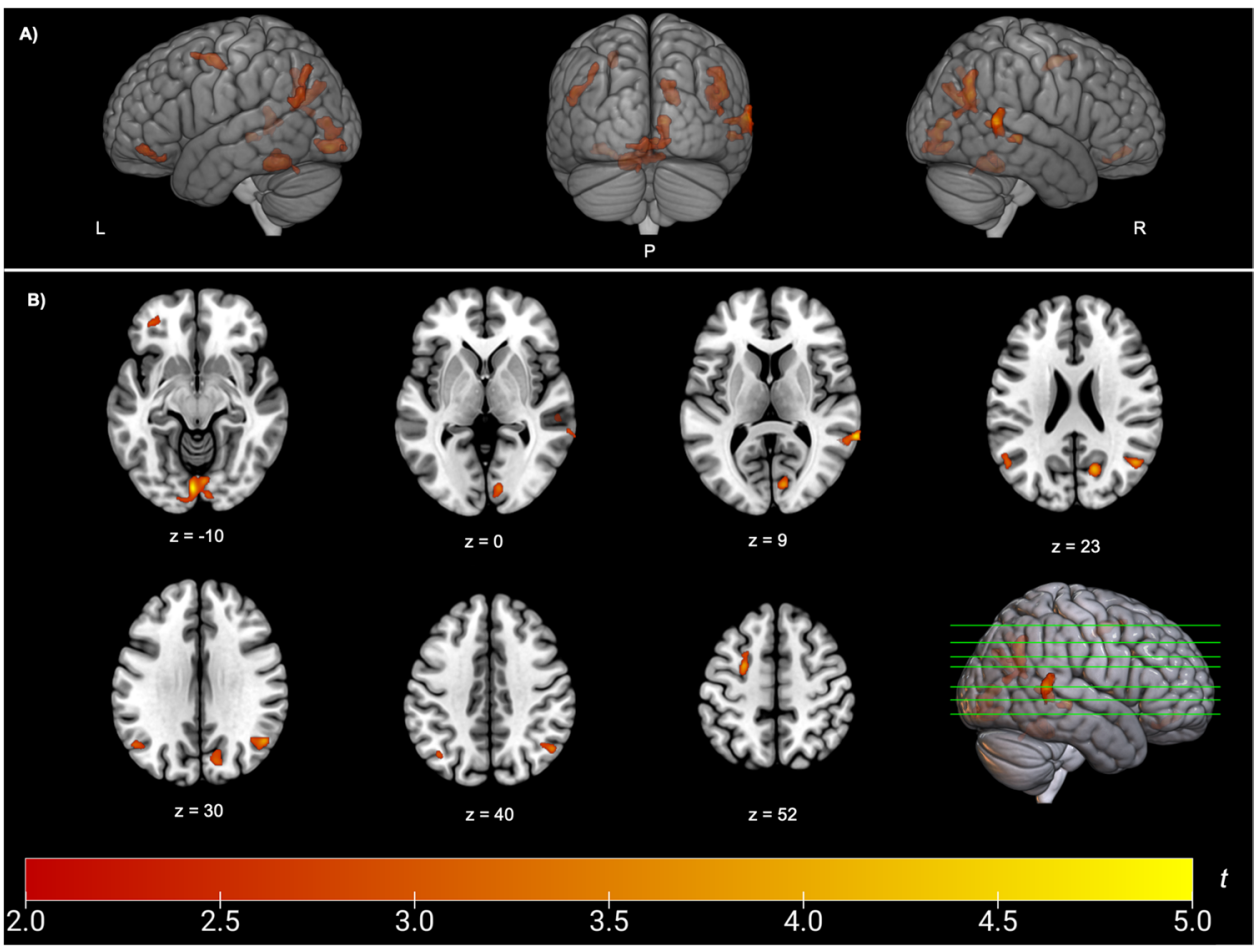
Table 3 Whole brain voxel-wise regression analysis

\begin{tabular}{|c|c|c|c|c|c|c|c|c|c|}
\hline \multicolumn{3}{|c|}{$\begin{array}{l}\text { Peak coordinates } \\
(\mathrm{MNI})\end{array}$} & \multirow[t]{2}{*}{ Anatomical region } & \multirow[t]{2}{*}{ Side } & \multicolumn{4}{|c|}{ Cluster level } & \multirow[b]{2}{*}{$T$} \\
\hline$X$ & $\mathrm{Y}$ & $\mathrm{Z}$ & & & FDR-corr & uncor & & cluster size & \\
\hline-26 & -1 & 52 & Middle frontal gyrus & $\mathrm{L}$ & .737 & .044 & $*$ & 448 & 3.93 \\
\hline-24 & 8 & 53 & Superior frontal gyrus & $\mathrm{L}$ & - & - & & - & 3.41 \\
\hline-27 & 39 & -15 & Middle frontal gyrus, orbital part & $\mathrm{L}$ & .737 & .043 & $*$ & 455 & 4.42 \\
\hline-22 & 32 & -19 & Middle frontal gyrus & $\mathrm{L}$ & - & - & & - & 3.21 \\
\hline 67 & -45 & 9 & Middle temporal gyrus & $\mathrm{R}$ & .290 & .009 & $* *$ & 841 & 4.88 \\
\hline 57 & -30 & -4 & Middle temporal gyrus & $\mathrm{R}$ & - & - & & - & 4.39 \\
\hline 59 & -49 & 7 & Superior temporal gyrus & $\mathrm{R}$ & - & - & & - & 3.83 \\
\hline-54 & -60 & 20 & Middle temporal gyrus & $\mathrm{L}$ & .571 & .026 & $*$ & 570 & 3.54 \\
\hline-38 & -69 & 45 & Inferior parietal lobule & $\mathrm{L}$ & - & - & & - & 3.29 \\
\hline-41 & -66 & 37 & Inferior parietal lobule & $\mathrm{L}$ & - & - & & - & 3.19 \\
\hline 50 & -62 & 26 & Angular gyrus & $\mathrm{R}$ & .948 & .002 & $* *$ & 1223 & 4.76 \\
\hline 50 & -63 & -36 & Tuber (Cerebellum) & $\mathrm{R}$ & - & - & & - & 4.10 \\
\hline 49 & -72 & 14 & Middle temporal gyrus & $\mathrm{R}$ & - & - & & - & 3.14 \\
\hline-4 & -85 & -10 & Calcarine sulcus & $\mathrm{L}$ & .060 & .001 & $* * *$ & 1594 & 5.27 \\
\hline 11 & -83 & 7 & Cuneus & $\mathrm{R}$ & - & - & & - & 4.31 \\
\hline 5 & -79 & -8 & Lingual gyrus & $\mathrm{R}$ & - & - & & - & 4.15 \\
\hline 16 & -67 & 23 & Precuneus & $\mathrm{R}$ & .309 & .012 & $*$ & 766 & 4.13 \\
\hline 12 & -78 & 31 & Cuneus & $\mathrm{R}$ & - & - & & - & 3.27 \\
\hline
\end{tabular}

Note. SCD-S was entered as dependent variable. Age, sex, UPDRS-III OFF-state, and BDI-II were used as covariates. FDR-corr. False Discovery Rate Correction; $L$ left; $M N I$ Montreal Neurological Institute; $R$ right

${ }^{*} p \leq .05 ; * * p \leq .01 ; * * * p \leq .001$

\section{Predictors of SCD}

Stepwise regression analyses with age, sex, education, UPDRS-III ON-state, BDI-II, PANDA, and the ROI value as predictors were conducted to identify predictors of SCD, indicated by the subscore SCD-S. The final model explained $53.7 \%$ (adjusted $R^{2}=50.3 \%$ ) of the variance in the SCD-S score with only two significant predictors: depressive symptoms and ROI metabolism value (for details, see Table 4). Increasing depressive symptoms as measured with the BDI-II ( $\beta=0.467, p=0.002)$ correlated with more SCD, whereas a decreased FDG-metabolism in the above-mentioned ROIs was related to more $\operatorname{SCD}(\beta=-0.442, p=0.003)$.
Table 4 Final model of the stepwise regression analysis

\begin{tabular}{|c|c|c|c|c|c|c|}
\hline Predictor & $B$ & SE & $\beta$ & $t$ & $p$ & \\
\hline \multicolumn{7}{|l|}{ Final model } \\
\hline BDI-II & .564 & .166 & .467 & 3.399 & .002 & $* *$ \\
\hline ROI & -.834 & .259 & -.442 & -3.222 & .003 & $* *$ \\
\hline \multicolumn{7}{|c|}{$\mathrm{F}(2,27)=15.667, p<0.001 ; R^{2}=.537 ;$ adjusted $R^{2}=.503$} \\
\hline \multicolumn{7}{|l|}{ Excluded variables } \\
\hline Age & & & .140 & 1.067 & .296 & \\
\hline Education & & & -.018 & -0.127 & .900 & \\
\hline Sex & & & -.154 & -1.158 & .257 & \\
\hline PANDA & & & -.189 & -1.430 & .165 & \\
\hline UPDRS-III ON & & & .196 & 1.524 & .140 & \\
\hline
\end{tabular}

Note. SCD-S (specific concerns) subscore was used as dependent variable in the regression model. Sex was dummy-coded with $0=$ male, $1=$ female. BDI-II Beck Depression Inventory 2; PANDA Parkinson Neuropsychometric Dementia Assessment; UPDRS-III Unified Parkinson Disease Rating Scale part 3; ROI region of interest

${ }^{*} p \leq 0.05 ; * * p \leq 0.01 ; * * * p \leq 0.001$ 


\section{Discussion}

This study on demographic and behavioral determinants as well as on neural correlates of SCD in patients with PD revealed (i) decreased levels of FDG-metabolism in middle frontal, middle temporal and occipital areas, as well as in the angular gyrus in patients with higher SCD-S scores, and (ii) higher levels of depressive symptoms and a reduced regional FDG-metabolism in fronto-medial, temporo-medial, and parieto-occipital regions to be predictive of SCD.

One strength of this study is the focus on the broader SCD construct targeting a spectrum of cognitive domains instead of restricted concepts like SCD in the memory domain. This recognizes that cognitive decline in $\mathrm{PD}$ is highly heterogeneous and not focused on memory impairment only [11-14]. So far, no extensively validated or commonly used tools to assess SCD exist [7], which is why we assessed relationships of the SCD-Q subscales with other questionnaires. Importantly, correlational analyses revealed an association of the subscore SCD-S that was further used in the analyses and self-rated questionnaires assessing SCD-related concepts. However, no correlation was found between SCD-S and informant-rated versions of the SCD-related questionnaires. This is congruent with the definition of SCD as a self-perceived phenomenon [1]. Furthermore, patients with and without SCD showed different levels of SCD and SCDrelated concepts in the expected direction, with the PD-SCD group reporting higher levels of subjective complaints than the control group across different assessments. Importantly, no significant differences were found in objective cognitive functioning, clinical, or sociodemographic variables between those two groups. Additionally, the exclusion of patients with objective cognitive deficits in our study cohort was based on the established level-II diagnostic criteria for PD-MCI [39] and precluded the confounding of SCD by objective cognitive deficits.

Our findings on functional neuroimaging correlates of SCD are congruent with prior neuroimaging studies on SCD in memory in $\mathrm{PD}[9,15,26]$ and $\mathrm{AD}[22]$. Especially the middle frontal and middle temporal areas are involved in executive functions and semantic processing [11, 12]. In combination with parieto-occipital areas, processing visuocognitive functions, these structures are also part of the attentional system [11, 12]. Therefore, the spectrum of neural correlates might reflect a pre-clinical stage of cognitive impairment in PD, which already mirrors the heterogeneity of objective cognitive impairment in PD, for example, as stated in the dual syndrome hypothesis [11, 12]. Further supporting this hypothesis, Huang et al. [41, 42] found FDGhypometabolism in the middle frontal gyrus and inferior parietal lobule in patients with PD-MCI, indicating that the pattern of hypometabolism we detected in PD-SCD might represent early PD-related pathological changes. Glucose hypometabolism was also found in parieto-occipital areas of the brain in patients with PD and objective cognitive dysfunctions [43]. Importantly, it has been shown previously that metabolic reductions in occipital brain areas herald the onset of PDD [44]. Furthermore, parieto-occipital hypometabolism is a prominent feature to discriminate Lewy-Bodydementia and PDD from AD [45, 46]. Overall, the broad spectrum of neuronal correlates of SCD in PD highlights the importance of assessing SCD as a broad construct not limited to perceived memory impairment.

Regional FDG-hypometabolism in fronto-medial, temporomedial, and parieto-occipital regions was also found to significantly predict SCD in PD within our stepwise regression analyses, which additionally investigated the influence of demographic, clinical, and neuropsychological predictors of SCD in PD. Next to FDG-metabolism, depressive symptoms emerged as an independent significant positive predictor of SCD in PD. Depressive symptoms have been identified as a reliable determinant of SCD in the general aging context before $[16,19,20]$. As Burmester et al. [16] discuss in their systematic review, depressive symptoms might even account for any observed relationship between objective cognitive functions and SCD. This in turn might explain the finding of global cognitive functioning assessed with the PANDA not being a significant predictor for SCD in our PD cohort. However, it can also be hypothesized that the phenomenon of depressive realism might account for the strong relationship of depressive symptoms and SCD, as the depressive symptomatology might lead to excessively negative or "skeptical" perceptions of one's cognitive state. In this context, the PANDA cognitive screening tool was probably not sensitive enough to reflect any associations of SCD with objective cognitive performance. Furthermore, it was previously shown that the relationship between subjective and objective cognitive performance is rather unreliable anyhow [47].

Demographic variables such as age, education, and sex were not identified as significant independent determinants of SCD in our cohort. In the general aging context, SCD were found to be more frequent in women than men and positively related to age and education [16]. In this context, however, for example, the role of age-related stereotypes and the perception of one's own health are discussed. In PD patients, general age-related stereotypes might be less important than perceived stereotypes associated with PD itself. However, PD motor impairment as assessed with the UPDRS-III did not emerge as an independent significant determinant of SCD either. Motor impairment was previously found to be a significant predictor for objective cognitive decline in PD, arguing that more severe motor impairment might indicate more severe neuro-pathological changes that in turn increase the risk for cognitive deficits 
[48]. However, our sample of patients with PD had no objective cognitive impairment and motor impairment was only mild. Therefore, the extent to which inferences from motor impairment to SCD were drawn was probably reduced. This hypothesis is strengthened by findings of the only study investigating determinants of SCD in the memory domain in PD so far [21]. In this study, motor impairment was a significant predictor of SCD in the memory domain in patients with PD-MCI, but not for patients without objective cognitive impairment, which is in agreement with our results.

One limitation is that the sample size was relatively small. However, our findings are supported by previous literature, which in turn supports our approach to measure SCD and implies that it is sensitive enough to detect effects in a relatively small sample. The major strength of this study, as already discussed above, was the operationalization of the SCD construct based on sound diagnostic criteria excluding objective cognitive impairment by level-II diagnostic criteria of PD-MCI [39] and using an elaborated SCD questionnaire assessing several cognitive domains and daily life situations. Furthermore, to the author's best knowledge, this analysis constitutes the first approach of combining research on neuronal and sociodemographic, clinical, and neuropsychological correlates of SCD in PD.

Summarizing, the present study strengthens that SCD may be an early manifestation of future cognitive decline and, more generally speaking, early pathological changes in PD. Objective, validated screening tools and diagnostic criteria for SCD in PD should be developed. We identified hypometabolism in fronto-medial, temporo-medial, and parieto-occipital regions as well as more depressive symptoms as independent determinants of SCD in PD. The early identification of vulnerability for future cognitive decline constitutes the basis for successful prevention and delaying the onset of objective cognitive decline. Furthermore, the better understanding of determinants of SCD might help to develop targeted treatment approaches in cognitive prevention, training, and rehabilitation.

Supplementary Information The online version contains supplementary material available at https://doi.org/10.1007/s10072-021-05734-w.

Funding Open Access funding enabled and organized by Projekt DEAL. This study received funding by the Cologne Fortune Program and by the German Research Foundation (Deutsche Forschungsgemeinschaft, DFG) (KFO219, Grant number EG350/1-1).

Data availability The datasets generated during and/or analyzed during the current study are available from the corresponding author on reasonable request.

Code availability Code used to analyze the data of the current study is available from the corresponding author on reasonable request.

\section{Declarations}

Ethics approval Both studies contributing data to the present analyses were approved by the ethics committee of the medical faculty of the University of Cologne, Germany (approval numbers 12-265 and 15-325).

Consent to participate Every subject provided informed consent to participate in the study. The authors assert that all procedures contributing to this work comply with the ethical standards of the relevant national and institutional committees on human experimentation and with the Helsinki Declaration of 1975, as revised in 2008.

Conflict of interest $\mathrm{AO}$ and $\mathrm{FK}$ do not declare any conflicts of interest. EK has received grants from the German Ministry of Education and Research, ParkinsonFonds Deutschland GmbH, the German Parkinson Society; honoraria from Oticon $\mathrm{GmbH}$, Hamburg, Germany; Lilly Pharma GmbH, Bad Homburg, Germany; Bernafon AG, Bern, Switzerland; Desitin GmbH, Hamburg, Germany; all outside the submitted work.

AG does not declare any conflicts of interest.

AD received grants from Siemens Healthineers, Life Molecular Imaging, GE Healthcare, AVID Radiopharmaceuticals; speaker honorary or payments in advisory boards from Siemens Healthineers, Sanofi, GE Healthcare, Stock: Siemens Healthineers; patents: patent pending for 18F-PSMA7 (PSMA PET imaging tracer); all outside the submitted work.

MT does not declare any conflicts of interest.

LT received payments as a consultant for Medtronic Inc. and Boston Scientific and received honoraria as a speaker on symposia sponsored by Medtronic, Boston Scientific, and Abbott.

FJ received in the last 12 months payments as a consultant for Abbvie In.c, Biogen, Roche Esai, AC immune, and Janssen; all outside the submitted work.

$\mathrm{CE}$ received in the last 12 months payments as a consultant for Abbvie Inc. CE received honoraria as a speaker from Abbvie Inc. and received payments as a consultant for Abbvie Inc., Bial Inc., and Philyra Inc.; all outside the submitted work.

FM does not declare any conflicts of interest.

Open Access This article is licensed under a Creative Commons Attribution 4.0 International License, which permits use, sharing, adaptation, distribution and reproduction in any medium or format, as long as you give appropriate credit to the original author(s) and the source, provide a link to the Creative Commons licence, and indicate if changes were made. The images or other third party material in this article are included in the article's Creative Commons licence, unless indicated otherwise in a credit line to the material. If material is not included in the article's Creative Commons licence and your intended use is not permitted by statutory regulation or exceeds the permitted use, you will need to obtain permission directly from the copyright holder. To view a copy of this licence, visit http://creativecommons.org/licenses/by/4.0/.

\section{References}

1. Jessen F, Amariglio RE, Van Boxtel M, Breteler M, Ceccaldi M, Chételat G, Dubois B, Dufouil C, Ellis KA, Van Der Flier WM (2014) A conceptual framework for research on subjective cognitive decline in preclinical Alzheimer's Disease. Alzheimers Dement 10:844-852

2. Jessen F, Wiese B, Bachmann C, Eifflaender-Gorfer S, Haller F, Kölsch H, Luck T, Mösch E, van den Bussche H, Wagner M 
(2010) Prediction of dementia by subjective memory impairment: effects of severity and temporal association with cognitive impairment. Arch Gen Psychiatry 67:414-422

3. Reisberg B, Shulman MB, Torossian C, Leng L, Zhu W (2010) Outcome over seven years of healthy adults with and without subjective cognitive impairment. Alzheimers Dement 6:11-24

4. Rabin LA, Smart CM, Crane PK, Amariglio RE, Berman LM, Boada M, Buckley RF, Chételat G, Dubois B, Ellis KA (2015) Subjective cognitive decline in older adults: an overview of selfreport measures used across 19 international research studies. J Alzheimers Dis 48:S63-S86

5. Molinuevo JL, Rabin LA, Amariglio R, Buckley R, Dubois B, Ellis KA, Ewers M, Hampel H, Klöppel S, Rami L (2017) Implementation of subjective cognitive decline criteria in research studies. Alzheimers Dement 13:296-311

6. Aarsland D, Creese B, Politis M, Chaudhuri KR, Weintraub D, Ballard C (2017) Cognitive decline in Parkinson disease. Nat Rev Neurol 13:217-231

7. Kjeldsen PL, Damholdt MF (2019) Subjective cognitive complaints in patients with Parkinson's disease. Acta Neurol Scand 140:375-389

8. Erro R, Santangelo G, Barone P, Picillo M, Amboni M, Longo K, Giordano F, Moccia M, Allocca R, Pellecchia MT (2014) Do subjective memory complaints herald the onset of mild cognitive impairment in Parkinson disease? J Geriatr Psychiatry Neurol 27:276-281

9. Hong JY, Yun HJ, Sunwoo MK, Ham JH, Lee J-M, Sohn YH, Lee $\mathrm{PH}$ (2014) Cognitive and cortical thinning patterns of subjective cognitive decline in patients with and without Parkinson's Disease. Parkinsonism Relat Disord 20:999-1003

10. Galtier I, Nieto A, Lorenzo JN, Barroso J (2019) Subjective cognitive decline and progression to dementia in Parkinson's disease: a long-term follow-up study. J Neurol 266:745-754

11. Kehagia AA, Barker RA, Robbins TW (2013) Cognitive impairment in Parkinson's disease: the dual syndrome hypothesis. Neurodegener Dis 11:79-92

12. Kehagia AA, Barker RA, Robbins TW (2010) Neuropsychological and clinical heterogeneity of cognitive impairment and dementia in patients with Parkinson's disease. Lancet Neurol 9:1200-1213

13. Litvan I, Aarsland D, Adler CH, Goldman JG, Kulisevsky J, Mollenhauer B, Rodriguez-Oroz MC, Tröster AI, Weintraub D (2011) MDS task force on mild cognitive impairment in Parkinson's disease: critical review of PD-MCI. Mov Disord 26:1814-1824

14. Muslimović D, Post B, Speelman JD, Schmand B (2005) Cognitive profile of patients with newly diagnosed Parkinson disease. Neurology 65:1239-1245

15. Hong JY, Lee JE, Sohn YH, Lee PH (2012) Neurocognitive and atrophic patterns in Parkinson's disease based on subjective memory complaints. J Neurol 259:1706-1712

16. Burmester B, Leathem J, Merrick P (2016) Subjective cognitive complaints and objective cognitive function in aging: a systematic review and meta-analysis of recent cross-sectional findings. Neuropsychol Rev 26:376-393

17. Hänninen T, Reinikainen KJ, Helkala EL, Koivisto K, Mykkänen L, Laakso M, Pyörälä K, Riekkinen PJ (1994) Subjective memory complaints and personality traits in normal elderly subjects. J Am Geriatr Soc 42:1-4

18. Slavin MJ, Brodaty H, Kochan NA, Crawford JD, Trollor JN, Draper B, Sachdev PS (2010) Prevalence and predictors of "subjective cognitive complaints" in the sydney memory and ageing study. Am J Geriatr Psychiatry 18:701-710

19. Montejo P, Montenegro M, Fernández MA, Maestú F (2011) Subjective memory complaints in the elderly: prevalence and influence of temporal orientation, depression and quality of life in a population-based study in the city of Madrid. Aging Ment Health $15: 85-96$
20. Schmand B, Jonker C, Geerlings MI, Lindeboom J (1997) Subjective memory complaints in the elderly: depressive symptoms and future dementia. Br J Psychiatry 171:373-376

21. Baschi R, Nicoletti A, Restivo V, Recca D, Zappia M, Monastero R (2018) Frequency and correlates of subjective memory complaints in Parkinson's disease with and without mild cognitive impairment: data from the Parkinson's disease cognitive impairment study. J Alzheimers Dis 63:1015-1024

22. Saykin A, Wishart H, Rabin L, Santulli R, Flashman L, West J, McHugh T, Mamourian A (2006) Older adults with cognitive complaints show brain atrophy similar to that of amnestic MCI. Neurology 67:834-842

23. Scheef L, Spottke A, Daerr M, Joe A, Striepens N, Kölsch H, Popp J, Daamen M, Gorris D, Heneka MT (2012) Glucose metabolism, gray matter structure, and memory decline in subjective memory impairment. Neurology 79:1332-1339

24. López-Sanz D, Bruña R, Garcés P, Martín-Buro MC, Walter S, Delgado ML, Montenegro M, López Higes R, Marcos A, Maestú F (2017) Functional connectivity disruption in subjective cognitive decline and mild cognitive impairment: a common pattern of alterations. Front Aging Neurosci 9:109

25. Viviano RP, Damoiseaux JS (2020) Functional neuroimaging in subjective cognitive decline: current status and a research path forward. Alzheimers Res Ther 12:1-18

26. Song I-U, Kim J-S, Chung S-W, Lee K-S, Oh J-K, Chung Y-A (2014) Early detection of subjective memory impairment in Parkinson's disease using cerebral perfusion SPECT. Bio-Med Mater Eng 24:3405-3410

27. Ruppert MC, Greuel A, Tahmasian M, Schwartz F, Stürmer S, Maier F, Hammes J, Tittgemeyer M, Timmermann L, Van Eimeren T (2020) Network degeneration in Parkinson's disease: multimodal imaging of nigro-striato-cortical dysfunction. Brain 143:944-959

28. Hammes J, Theis H, Giehl K, Hoenig MC, Greuel A, Tittgemeyer M, Timmermann L, Fink GR, Drzezga A, Eggers C (2019) Dopamine metabolism of the nucleus accumbens and fronto-striatal connectivity modulate impulse control. Brain 142:733-743

29. Kalbe E, Bintener C, Ophey A, Reuter C, Göbel S, Klöters S, Baller G, Kessler J (2018) Computerized cognitive training in healthy older adults: Baseline cognitive level and subjective cognitive concerns predict training outcome. Health 10:20-55

30. Jessen F, Wiese B, Bickel H, Eiffländer-Gorfer S, Fuchs A, Kaduszkiewicz H, Köhler M, Luck T, Mösch E, Pentzek M (2011) Prediction of dementia in primary care patients. PLoS ONE 6:1-10

31. Tomlinson CL, Stowe R, Patel S, Rick C, Gray R, Clarke CE (2010) Systematic review of levodopa dose equivalency reporting in Parkinson's disease. Mov Disord 25:2649-2653

32. Fahn S, Elton R, Members of the UPDRS Development Committee (1987) Unified Parkinson's disease rating scale In Recent Developments in Parkinson's Disease, Fahn S, Marsden CD, Goldstein M, Calne DB, eds. Macmillan Healthcare Information, Florham Park, NJ, 153-163

33. Beck AT, Steer RA, Brown GK (1996) Beck depression inventoryII. San Antonio 78:490-498

34. Farias ST, Mungas D, Reed BR, Cahn-Weiner D, Jagust W, Baynes K, DeCarli C (2008) The measurement of everyday cognition (ECog): scale development and psychometric properties. Neuropsychology 22:531

35. Broadbent DE, Cooper PF, FitzGerald P, Parkes KR (1982) The cognitive failures questionnaire (CFQ) and its correlates. Br J Clin Psychol 21:1-16

36. Wilson B, Alderman N, Burgess P, Emslie H, Evans J (1996) Behavioural assessment of the dysexecutive syndrome (BADS), Thames Valley Test Company. Bury St. Edmunds, England 
37. Folstein MF, Folstein SE, McHugh PR (1975) "Mini-mental state": a practical method for grading the cognitive state of patients for the clinician. J Psychiatr Res 12:189-198

38. Kalbe E, Calabrese P, Kohn N, Hilker R, Riedel O, Wittchen H-U, Dodel R, Otto J, Ebersbach G, Kessler J (2008) Screening for cognitive deficits in Parkinson's disease with the Parkinson neuropsychometric dementia assessment (PANDA) instrument. Parkinsonism Relat Disord 14:93-101

39. Litvan I, Goldman JG, Tröster AI, Schmand BA, Weintraub D, Petersen RC, Mollenhauer B, Adler CH, Marder K, WilliamsGray CH (2012) Diagnostic criteria for mild cognitive impairment in Parkinson's disease: movement disorder society task force guidelines. Mov Disord 27:349-356

40. Greuel A, Trezzi JP, Glaab E, Ruppert MC, Maier F, Jäger C, Hodak Z, Lohmann K, Ma Y, Eidelberg D (2020) GBA Variants in Parkinson's disease: clinical, metabolomic, and multimodal neuroimaging phenotypes. Mov Disord

41. Huang C, Tang C, Feigin A, Lesser M, Ma Y, Pourfar M, Dhawan V, Eidelberg D (2007) Changes in network activity with the progression of Parkinson's disease. Brain 130:1834-1846

42. Huang C, Mattis P, Perrine K, Brown N, Dhawan V, Eidelberg D (2008) Metabolic abnormalities associated with mild cognitive impairment in Parkinson disease. Neurology 70:1470-1477

43. Garcia-Garcia D, Clavero P, Salas CG, Lamet I, Arbizu J, Gonzalez-Redondo R, Obeso JA, Rodriguez-Oroz MC (2012) Posterior parietooccipital hypometabolism may differentiate mild cognitive impairment from dementia in Parkinson's disease. Eur J Nucl Med Mol Imaging 39:1767-1777
44. Bohnen NI, Koeppe RA, Minoshima S, Giordani B, Albin RL, Frey KA, Kuhl DE (2011) Cerebral glucose metabolic features of Parkinson disease and incident dementia: longitudinal study. J Nucl Med 52:848-855

45. Imamura $T$, Ishii $K$, Hirono N, Hashimoto M, Tanimukai S, Kazui H, Hanihara T, Sasaki M, Mori E (2001) Occipital glucose metabolism in dementia with lewy bodies with and without Parkinsonism: a study using positron emission tomography. Dement Geriatr Cogn Disord 12:194-197

46. Minoshima S, Foster NL, Sima AA, Frey KA, Albin RL, Kuhl DE (2001) Alzheimer's disease versus dementia with Lewy bodies: cerebral metabolic distinction with autopsy confirmation. Ann Neurol 50:358-365

47. Crumley JJ, Stetler CA, Horhota M (2014) Examining the relationship between subjective and objective memory performance in older adults: a meta-analysis. Psychol Aging 29:250

48. Hu M, Szewczyk-Królikowski K, Tomlinson P, Nithi K, Rolinski M, Murray C, Talbot K, Ebmeier KP, Mackay CE, Ben-Shlomo Y (2014) Predictors of cognitive impairment in an early stage Parkinson's disease cohort. Mov Disord 29:351-359

49. Emre M (2003) Dementia associated with Parkinson's disease. Lancet Neurol 2:229-237

Publisher's note Springer Nature remains neutral with regard to jurisdictional claims in published maps and institutional affiliations. 